MATEC Web of Conferences 51, 01005 (2016)

DOI: $10.1051 /$ matecconf/20165101005

(C) Owned by the authors, published by EDP Sciences, 2016

\title{
Electro-Mechanical Response and Engineering Properties of Piezocomposite with Imperfect Interface
}

\author{
Rattanan Tippayaphalapholgul ${ }^{1}$ and Yasothorn Sapsathiarn ${ }^{1, a}$ \\ 1Department of Civil and Environmental Engineering, Faculty of Engineering, MahidolUniversity, Thailand
}

\begin{abstract}
Composites of piezoelectric materials are widely use in practical applications such as nondestructive testing devices, smart adaptive structures and medical devices. A thorough understanding of coupled electro-elastic response and properties of piezocomposite are crucial for the development and design of piezoelectric composite materials used in advanced applications. The micromechanics analysis is employed in this paper to determine the response and engineering properties of the piezocomposite. A mechanical imperfect interface bonding between piezoelectric inclusion and polymer matrix is taken into consideration in the analysis. The micromechanics analysis is based on the Boundary Element Method (BEM) together with the periodic micro-field micromechanics theory. A selected set of numerical results is presented to investigate the influence of volume ratio and interface bonding condition on effective piezocomposite material coefficients and portray basic features of coupled electroelastic response within the domain of piezocomposite unit cell.
\end{abstract}

\section{Introduction}

Piezoelectric materials have been used in the development of a variety of electro-mechanical devices due to their inherent electro-mechanical coupling phenomenon; that is, they produce an electric field when deformed under a mechanical stress (direct piezoelectric effect) and, conversely, undergo deformation when subjected to an electric field (converse piezoelectric effect) $[1,2]$. Several issues related to properties of monolithic piezoelectric elements, for example, low fracture strength, high stiffness and difficulty in producing complex shapes, lead to the development of composites of piezoelectric materials to improve overall properties which are suitable for practical applications. Figure 1(a) shows a composite of piezoelectric material and polymer matrix. The soft polymer matrix supports the brittle piezoelectric fibers and also offers more conformability of piezocomposite.

An understanding of coupled electroelastic behavior of piezoelectric composite materials and their properties is critical for an effective and reliable design of electro-mechanical devices based on piezocomposites. The response of piezoelectric composite materials is governed by several parameters, such as the fiber-to-matrix volume ratio, the properties of the fiber, the surrounding matrix material, and the interphase layer between piezoelectric fibers and the matrix [3-5]. Properties of piezocomposites can be globally represented by a homogeneous medium with anisotropic properties.Micromechanics model can provide the relations between the microstructure and the overall

${ }^{\mathrm{a} C}$ Corresponding author : Yasothorn.sap@mahidol.ac.th 
or effective properties of composites based on the known properties of each material phases in the composites.

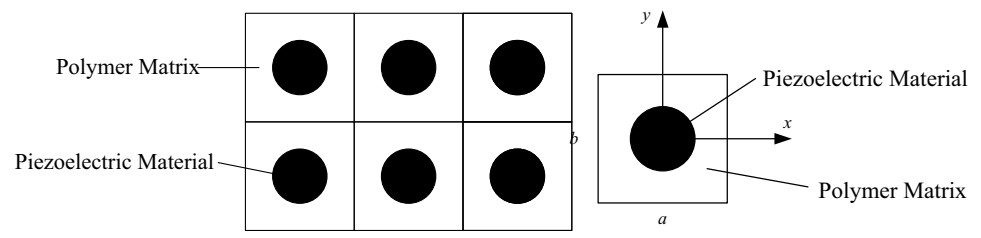

(a) (b)

Figure 1.(a) Schematic of piezoelectric composite material; (b)Representative Volume Element (RVE)

Several researchers have employed a variety of analytical and numerical techniques to predict the effective electromechanical moduli of piezocomposites [6-9]. Most of studies on responses and properties of composite materials appeared in the literature assume that the bonding between the fiber/inclusion and the matrix phase are perfect. In fact, there is a thin interphase layer between phases leading to imperfect bonding along the interface between the fiber/inclusion and matrix phases. Assumptions are usually employed in the modeling of the interphasial layer due to the structure of interphase layer is very complicated. Among others, a spring-factor model is the most widely used in the analysis. The model assumes that the displacement jump across the interface to be proportional to the corresponding interfacial stresses [10]. The spring-factor model for imperfect interface has been employed by [11] and [12] in the analysis of elastic fiber-reinforced composites.

The micromechanics model for composites of piezoelectric materials and polymer matrix is developed in this paper. The model is based on the periodic microfield micromechanics theory and the boundary element method (BEM). A selected set of numerical results are presented to portray the essential characteristics of electroelastic fields and effective properties of a piezocomposite

\section{Theoretical consideration for BEM-based micromechanics analysis of piezocomposite}

The composite of piezoelectric materials (fiber/inclusion) embedded in a polymeric matrix material is considered in this paper (Figure 1). An efficient methodology of micromechanics analysis for determining the effective properties of piezoelectric composite materials based on the boundary element method (BEM) and the periodic microfield approach is developed and explained in the subsequent sections. The static electroelastic response of a piezoelectric material is governed by the following equations expressed using the standard indicial notation [13]; $\sigma_{i j, j}+F_{i}=0$ and $D_{i, i}=\rho_{e}$, where $\sigma_{i j}(i, j=x, y, z), D_{i}, F_{i}$ and $\rho_{e}$ denote the stress tensor, electric displacement and body force in the $i$-direction, and a body charge, respectively.

The constitutive equations, strain-displacement and electric field-potential relations can be expressed as $\sigma_{i j}=c_{i j k l} \epsilon_{k l}-e_{k i j} E_{k}, D_{i}=e_{i k l} \epsilon_{k l}+\varepsilon_{i k} E_{k}, \epsilon_{i j}=\frac{1}{2}\left(u_{i, j}+u_{j, i}\right)$ and $E_{i}=-\phi_{i}$, where $u_{i}, \epsilon_{i j}$, $E_{i}$ and $\phi$ denote the displacement in the $i$-direction, strain tensor, electric field in the $i$-direction and electric potential, respectively; and $c_{i j k l}, e_{k i j}$ and $\varepsilon_{i k}$ denote elastic, piezoelectric and dielectric permittivity tensors, respectively. In the case of ideal elastic materials and rigid dielectrics, $e_{k i j}=0$. It can be also proved that $e_{k i j}=0$ in the case of an isotropic material and the piezoelectric effect does not exist.

The number of independent coefficients depends on the symmetry of the material. Piezoelectric solids used in most practical applications are either crystal with hexagonal symmetry or polarized 
ceramics. Constitutive equations for a piezoelectric solid having hexagonal symmetry about the $z-$ axis undergoing plane strain deformations $\left(\epsilon_{y y}, \epsilon_{x y}, \epsilon_{z y}=0, E_{y}=0\right)$ can be expressed as

$\sigma_{x x}=c_{11} \epsilon_{x x}+c_{13} \epsilon_{z z}-e_{31} E_{z} ; \sigma_{z z}=c_{13} \epsilon_{x x}+c_{33} \epsilon_{z z}-e_{33} E_{z} ; \sigma_{z x}=2 c_{44} \epsilon_{x z}-e_{15} E_{x}$ (1a)

$D_{x}=2 e_{15} \epsilon_{x z}+\varepsilon_{11} E_{x} ; D_{z}=e_{31} \epsilon_{x x}+e_{33} \epsilon_{z z}-\varepsilon_{33} E_{z}(1 \mathrm{~b})$

where $c_{11}, c_{13}, c_{33}$ and $c_{44}$ denote elastic moduli; $e_{31}, e_{33}$ and $e_{15}$ denote piezoelectric constants and $\varepsilon_{11}, \varepsilon_{33}$ denote the dielectric permittivity.

Based on the micromechanics theory, the piezoelectric composite is a homogeneous orthotropic material. The micromechanical analysis of composite material based on the periodic micro-field approach has a key assumption that the composite material consists of periodically repeated microstructures.

The behavior and properties of the microstructure can be identified by a Representative Volume Element (RVE) which or unit cell which can be isolated and is in a state of equilibrium. The concept of periodic RVE of a piezoelectric composite is schematically represented in Figure 1(b). The RVE has the overall dimensions $a \times b$. If the ratio $a / b=1$, then the effective properties in both transverse direction of the composite are identical. Effective material coefficients of piezocomposites can be determined by analysis of a periodic RVE with appropriate boundary conditions. The macroscopic constitutive relation, which defines the effective material properties $c_{i j}^{\text {eff }}, e_{i j}^{\text {eff }}$ and $\varepsilon_{i j}^{\text {eff }}$ of piezocomposites, is represented in the form given by (1) which can be determined by consideringthe macro-stress and macro-strain $\bar{\sigma}_{i j}$ and $\bar{\varepsilon}_{i j}$ of the composite [8]. According to the micromechanics theory, the macro-stress $\left(\bar{\sigma}_{i j}\right)$ and macro-strain $\left(\bar{\epsilon}_{i j}\right)$ of composites can be defined as the volume average in an RVE as $\bar{\sigma}_{i j}=(1 / V) \int_{\Omega} \sigma_{i j} d \Omega ; \bar{\epsilon}_{i j}=(1 / V) \int_{\Omega} \epsilon_{i j} d \Omega$ where $V$ is the volume of an RVE in the domain $\Omega$. Similarly, the average electric displacement and electric field can be defined as $\bar{D}_{i}=(1 / V) \int_{\Omega} D_{i} d \Omega ; \bar{E}_{i}=(1 / V) \int_{\Omega} E_{i} d \Omega$.

The volume average of field variables can be related to the boundary field variables of the RVE by using Gauss theorem.Therefore, the boundary field variables of the RVE can be used in the calculation of the effective composite material properties and, with the boundary element analysis, the calculation involves discretization of the boundary of a microstructure only. Therefore, the BEMbased micromechanics model is very suitable for performing the calculation of effective composite material properties.

In this study, the mechanically imperfect fiber-matrix bonding is represented by a spring-factor model, which assumes that the displacement jumps along the interface are related to the interface stresses through a spring-factor parameter. The interface compatibility and continuity conditions between the fiber and matrix are given by $\tau_{i}^{f}+\tau_{i}^{m}=0$ and $u_{i}^{f}-u_{i}^{m}=m_{i} \tau_{i}^{f}$ where $m_{i}$ denotes the spring-factor parameter and superscript " $\mathrm{f}$ " and " $\mathrm{m}$ " are used to identify the variables associated with the fiber and matrix phases respectively. It is noted that for the limiting case of perfect fiber-matrix bonding: $m \rightarrow 0$ and $u_{i}^{f}=u_{i}^{m}$.

\section{Numerical results and discussion}

A computer code based on the boundary element method (BEM) and micromechanics theory outlined in the preceding sections has been developed. The accuracy of the present formulation is first verified through the comparison with existing solutions reported in the literature. The results of comparison are not presented for brevity. Excellent agreement between the present solutions and existing solutions is noted confirming the accuracy and validation of the present formulation.

A selected set of numerical solutions is presented in Figure 2-4 to demonstrate the basic features of electroelastic responses within a domain of piezoelectric composite material and the influence of various parameters, e.g., piezoelectric volume fraction and interface bonding condition, to the 
effective properties of piezoelectric composite material piezocomposite. Piezocomposite in which the elastic polymer matrix is periodically embedded with $\mathrm{BaTiO}_{3}$ piezoelectric material is considered in the numerical example. The material properties of $\mathrm{BaTiO}_{3}$ are: $c_{11}=15.0 \times 10^{10} \mathrm{~N} / \mathrm{m}^{2}, c_{33}=14.6 \times 10^{10}$ $\mathrm{N} / \mathrm{m}^{2}, c_{13}=6.6 \times 10^{10} \mathrm{~N} / \mathrm{m}^{2}, c_{44}=4.4 \times 10^{10} \mathrm{~N} / \mathrm{m}^{2}, e_{15}=11.4 \mathrm{C} / \mathrm{m}^{2}, e_{31}=-4.35 \mathrm{C} / \mathrm{m}^{2}, e_{33}=17.5 \mathrm{C} / \mathrm{m}^{2}$, $d_{11}=9.87 \times 10^{-9} \mathrm{~F} / \mathrm{m}, d_{33}=11.15 \times 10^{-9} \mathrm{~F} / \mathrm{m}$; and the polymer material properties are: $c_{11}=1.49 \times 10^{10}$ $\mathrm{N} / \mathrm{m}^{2}, c_{33}=4.72 \times 10^{10} \mathrm{~N} / \mathrm{m}^{2}, c_{13}=0.52 \times 10^{10} \mathrm{~N} / \mathrm{m}^{2}, c_{44}=0.47 \times 10^{10} \mathrm{~N} / \mathrm{m}^{2}$.

The boundary conditions along the outer surfaces of the RVE of piezocomposite are given by

Surface $x / a=-0.5: u_{x}=0.0 \mathrm{~m}, t_{z}=0.0 \mathrm{~Pa}, q=0.0 \mathrm{C} / \mathrm{m}^{2}$

Surface $x / a=0.5: u_{x}=0.0 \mathrm{~m}, t_{z}=0.0 \mathrm{~Pa}, q=0.0 \mathrm{C} / \mathrm{m}^{2}$

Surface $z / a=-0.5: u_{z}=0.0 \mathrm{~m}, t_{x}=0.0 \mathrm{~Pa}, \phi=0.0$

Surface $z / a=0.5: u_{z}=\delta, t_{x}=0.0 \mathrm{~Pa}, q=0.0 \mathrm{C} / \mathrm{m}^{2}$
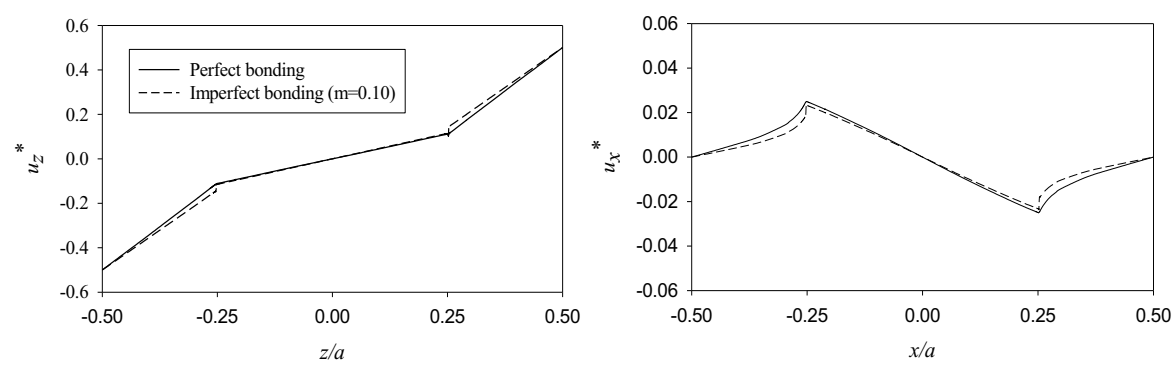

(a) Profile of $u_{z}^{*}$ along the $\mathrm{z}$-axis $(\mathrm{x}=0.0)(\mathrm{b})$ Profile of $u_{x}^{*}$ along the $\mathrm{x}$-axis $(\mathrm{z}=0.0)$

Figure 2. Nondimensionaldisplacements (a) $u_{z}^{*}$ along the $\mathrm{z}$-axis $(\mathrm{x}=0.0)$; (b) $u_{x}^{*}$ along the $\mathrm{x}$-axis $(\mathrm{z}=0.0)$

Figure 2 present profiles of the nondimensional displacements, $u_{z}^{*}=u_{z} / \delta$ along the z-axis $(\mathrm{x}=0.0)$ in Figure 2(a) and $u_{x}^{*}=u_{x} / \delta$ along the $\mathrm{x}$-axis $(\mathrm{z}=0.0)$ in Figure2(b) respectively, where $\delta$ denotes the prescribed displacement of the piezocomposite unit cell in z-direction. The fiber-matrix volume ratio considered in the present numerical example is $0.2\left(V_{f}=0.2\right)$. The results are presented in Figure2 for both perfect (solid line) and imperfect (dashed lined) interface bonding conditions between piezoelectric fiber/inclusion and polymer matrix. It can be seen in Figure 2(a) that all of the displacement curves are increasing functions (the slope is positive) indicating that the unit cell is extended with tensile strain along the z-axis under prescribed tension displacement in the $\mathrm{z}$ direction. It should be observed from the material properties that polymer matrix is less stiff than those of piezoelectric inclusion leading to a higher tensile strain as shown in Figure 2(a).

The corresponding contraction of piezoelectric material along the transverse direction ( $\mathrm{x}$-axis) for piezocomposite is presented in Figure 2(b). Due to the prescribed zero displacement in transverse direction of the RVE, the polymer matrix expands while the piezoelectric phase contracts. The influence of imperfect interface bonding condition on displacement profiles of piezocomposite unit cell can also be observed in Figure 2(a) and 2(b). The presence of imperfect interface bonding generally causes a lower strain in the unit cell for both piezoelectric and polymer phases. Moreover, a jump in displacements at the inclusion-matrix interface is noted in both figures.

Figure 3 shows the distribution of nondimensional stress $\left(\sigma_{z z}^{*}=\sigma_{z z} a / c_{33} \delta\right)$ and electric displacement $\left(D_{z}^{*}=D_{z} a / e_{33} \delta\right)$ within the unit cell of piezoelectric composite material in Figure 3(a) and Figure 3(b) respectively. It can be observed in Figure 3(a) and 3(b) that the stress and electric displacement contour are continuous over the domain of piezocomposite. Also, it can be seen in Figure 3(a) that the entire domain of piezoelectric composite material is under tensile stress with high concentration of $\sigma_{z z}^{*}$ stress induced over the central region over the piezoelectric inclusion. This is due 
to the presence of high stiffness piezoelectric inclusion at the center of the unit cell. In addition, due to symmetric loading and geometry of the unit cell, the symmetry of both stress $\sigma_{z z}^{*}$ (Figure 3(a)) and electric displacement $D_{z}^{*}$ distribution (Figure 3(b)) is noted.
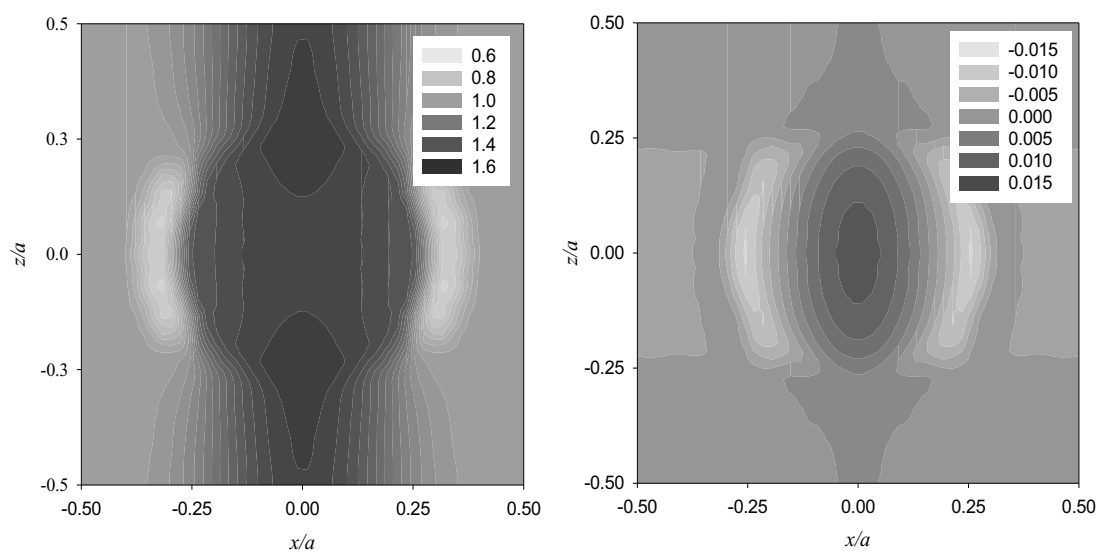

(a) Distribution of $\sigma_{z z}^{*}$ (b) Distribution of $D_{z}^{*}$

Figure 3. Distribution of (a) stress $\sigma_{z z}^{*}$ and (b) electric displacement $D_{z}^{*}$ within the unit cell of piezocomposite
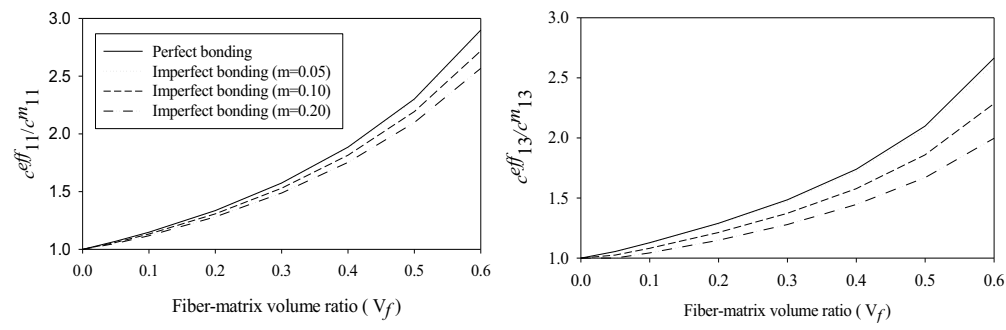

(a) $c_{11}^{\text {eff }} / c_{11}^{m}$ (b) $c_{13}^{e f f} / c_{13}^{m}$

Figure 4. Variation of effective coefficients of piezoelectric composite (a) $c_{11}^{\text {eff }} / c_{11}^{m}$; (b) $c_{13}^{\text {eff }} / c_{13}^{m}$

The variation of overall or effective material coefficients of the piezoelectric composite material with the volume fraction of the fiber $\left(V_{f}\right)$ are presented in Figure 4. Effective coefficients of material are calculated for the piezoelectric volume fraction in a range from 0.0 to 0.6 and the spring parameters $\mathrm{m}$ is set to be $0.01,0.1$ and 0.2 . As expected, the effective coefficients of piezocomposites depend on the fiber volume fraction and the interface bonding condition as can be seen in Figure 4(a) and 4(b). The nondimensional effective material coefficients dimensional $c_{11}^{e f f} / c_{11}^{m}$ effective material coefficients (Figure 4(a)) and $c_{13}^{\text {eff }} / c_{13}^{m}$ (Figure4(b)) has a unit magnitude at $V_{f}=0$. For the given material properties used in the numerical example, $\mathrm{BaTiO}_{3}$ piezoelectric material is stiffer than the polymer matrix. Therefore, when the fiber volume fraction increases, it leads to increasing values of effective composite material coefficients (see Figure4(a) and 4(b)). Nevertheless, it should be noted that the effective coefficients of the composite materials are not linearly increase with increasing piezoelectric volume fraction. Moreover, the numerical results in Figure 4 show that effective composite material properties do not only depend on the material properties of each phase in the composite but also depend on the bonding condition at the interface which can be interpreted from the value of the spring-factor parameter (m). In particular, it can be seen in Figure4(a) and 4(b) that the effective composite material coefficients $c_{11}^{\text {eff }} / c_{11}^{m}$ and $c_{13}^{\text {eff }} / c_{13}^{m}$ decrease for the case of a weaker interface bonding (higher value of $\mathrm{m}$ ) between piezoelectric material and polymer matrix. 


\section{Conclusion}

In this paper, a micromechanics model is developed and employed to study the influence of imperfect interface on effective piezoelectric composite material properties. The micromechanics analysis is based on the Boundary Element Method (BEM) together with the periodic micro-field micromechanics theory. The coupled electroelastic responses within the domain of piezoelectric composite material are also presented to portray basic features of piezocomposite unit cell. A mechanical imperfect interface bonding between piezoelectric inclusion and polymer matrix is also taken into consideration in the present analysis. The results from the numerical study show that the effective piezocomposite properties depend on the fiber-matrix volume fraction and interface bonding condition. The variation of the effective coefficient are non-linear with piezoelectric volume fraction.

The entire unit cell is stretched in the direction of prescribed displacement and stress concentration of stress is found over the central region. The distributions of nondimensional stress and electric displacement are continuous and symmetric due to symmetric loading and geometry of the unit cell considered. The numerical results presented in this paper provides a thorough understanding of coupled electro-elastic response within a unit cell and effective materials properties of piezocomposite which are useful for the design of piezocomposite-based electro-mechanical devices for advanced applications.

\section{Acknowledgements}

The work presented in this paper is supported by Mahidol University and partially supported by Graduate Studies of Mahidol University Alumni Association.

\section{References}

1. N.W.Hagood,A. A.Bent,Proc 43th AIAA/ASME, paper 93-1717(1993).

2. L. J. Nelson,Mater. Sci. Technol., 18, 1245(2002).

3. Y.Sapsathiarn, T.Senjuntichai,R.K.N.D. Rajapakse,Composites Part B: Engineering, 39, 1114(2008).

4. Sapsathiarn Y., Senjuntichai T. and Rajapakse R.K.N.D., Composites Part B: Engineering,43(5), 2257 (2012).

5. Sapsathiarn Y., Singh Y. ,Rajapakse R.K.N.D. and Mumford D. ,ActaMechanica, 225, 2943 (2014)

6. Kar-Gupta R, Venkatesh T. Acta Mater, 55, 1093 (2007)

7. Berger, H., Kari, S., Gabbert, U., Rodriguez-Ramos, R., Bravo-Castillero, J., Guinovart-Diaz, R., Sabina, F. J., Maugin, G. A., Smart Materials and Structures, 15(2), 451 (2006).

8. Poizat, C., and Sester, M.,Comput. Mater. Sci.,16, 89-97(1999).

9. Rajapakse, R. K. N. D., and Chen, Y., IEEE Trans. Ultrason. Ferroelect. Freq. Contr., 55, 1847 (2008).

10. Hammamia, H., Arousb, M., Lagachea, M., Kallel, A.Composites A, 37, 1 (2006).

11. Nairn, J.A. and Liu, Y.C., Int. J. Solids Structures,34, 1255 (1996).

12. Lenci, S. and Menditto, G., Int. J. Solids Structures.,37, 4239 (2000).

13. Parton, V.Z., \&Kudryavtsev, B.A. Electromagnetoelasticity: Piezoelectrics and Electrically Conductive Solids(Gordon and Breach Science,1988). 